\title{
Interlayer spin-singlet pairing induced by magnetic interactions in an antiferromagnetic superconductor
}

\author{
Hiroshi Shimahara \\ Department of Quantum Matter Science, ADSM, \\ Hiroshima University, Higashi-Hiroshima 739-8530, Japan
}

(Dated: Received 11 July 2004)

\begin{abstract}
It is shown that interlayer spin-singlet Cooper pairing is induced by magnetic interactions in a metallic antiferromagnet of stacked conductive layers in which each layer is ferromagnetically polarized and they order antiferromagnetically in stacking direction. As a result, the antiferromagnetic long-range order and superconductivity coexist at low temperatures. It is shown that $T_{A F}>T_{c}$ except for in a very limited parameter region unless $T_{A F}=0$, where $T_{A F}$ and $T_{c}$ denote the antiferromagnetic and superconducting transition temperatures, respectively. It is found that the exchange field caused by the spontaneous staggered magnetization does not affect superconductivity at all, even if it is very large. The resultant superconducting order parameter has a horizontal line node, and is isotropic in spin space in spite of the anisotropy of the background magnetic order. We discuss the possible relevance of the present mechanism to the antiferromagnetic heavy fermion superconductors $\mathrm{UPd}_{2} \mathrm{Al}_{3}$ and $\mathrm{CePt}_{3} \mathrm{Si}$.

PACS numbers: $\quad 74.20 .-\mathrm{z}, 74.20 . \mathrm{Mn}, 74.20 . \mathrm{Rp} 74.25 . \mathrm{Ha}$
\end{abstract}

In this paper, we show that interlayer spin-singlet Cooper pairing is induced by magnetic interactions in a certain kind of metallic antiferromagnet. We consider a layered system of itinerant electrons in which each layer is ferromagnetically polarized but the majority-spin alternates in stacking direction. Therefore, the magetic order is characterized by the wave vector $\boldsymbol{Q}=(0,0, \pi / c)$, where we have assumed the $a$ and $b$ crystal axes to be parallel to the layers, and the $c$-axis in the stacking direction, and $c$ denotes the $c$-axis lattice constant. It is also shown that the exchange field caused by spontaneous staggered magnetization does not influence superconductivity, however large it is.

The heavy fermion superconductors, such as $\mathrm{UPd}_{2} \mathrm{Al}_{3}$ and $\mathrm{CePt}_{3} \mathrm{Si}$, can be candidates of the present mechanism. The antiferromagnetic long-range order is considered to be characterized by the wave vector $\boldsymbol{Q}=$ $(0,0, \pi / c)$, both in $\mathrm{UPd}_{2} \mathrm{Al}_{3}$ [1] and in $\mathrm{CePt}_{3} \mathrm{Si}$ [2]. Superconducting transitions have been observed at $T_{c}=$ $2.0 \mathrm{~K}$ and $0.7 \mathrm{~K}$, below the antiferromagnetic transition temperatures $T_{A F}=14.3 \mathrm{~K}$ and $2.2 \mathrm{~K}$, in $\mathrm{UPd}_{2} \mathrm{Al}_{3}[3$ and $\mathrm{CePt}_{3} \mathrm{Si}$ [4, respectively. It has been suggested that the magnetic moment is large, i.e., $0.85 \mu_{B} / \mathrm{U}$, in $\mathrm{UPd}_{2} \mathrm{Al}_{3}$ [3] , but small, i.e., $0.16 \mu_{B} / \mathrm{Ce}$, in $\mathrm{CePt}_{3} \mathrm{Si}$ [2].

The order parameter of interlayer spin-singlet pairing has a horizontal line node. This also agrees with the experimental results in the compound $\mathrm{UPd}_{2} \mathrm{Al}_{3}$. The existence of the line node is suggested by the nuclear magnetic resonance (NMR) measurement [5], in which the Hebel-Slichter peak was absent, and $T_{1}^{-1} \propto T^{3}$ was observed. The singlet state is supported by the presence of NMR Knight shift [5], and the Pauli limited upper critical field [6, 7]. In recent angle resolved magnetothermal transport measurements [8], two-fold oscillation in the rotation perpendicular to the $a b$ plane was observed, while no oscillation was observed in rotation in the $a b$ plane. These experimental results are reproduced by the order parameter of the form of $\Delta(\boldsymbol{k})=\Delta_{0} \cos \left(k_{z} c\right)$.

Coexistence of superconductivity and magnetism has been studied in various models by many authors 9,10 , 11]. In particular, spin singlet superconductivity in the presence of ferromagnetic layers has been studied by many authors [9]. In the models examined in those papers, superconductivity occurs in a subsystem different from the magnetic layers. In contrast, we examine a model in which superconductivity occurs in electrons on magnetic layers in the present model. Kopaev also studied superconductivity when only magnetic electrons are present [10], although it exists only in the vicinity of the domain wall.

The magnetic structure mentioned above can be modeled most simply by the Hamiltonian

$$
H=H_{0}+H_{U}+H_{J}
$$

with the kinetic energy term

$$
H_{0}=\sum_{\boldsymbol{k} \sigma} \xi(\boldsymbol{k}) c_{\boldsymbol{k} \sigma}^{\dagger} c_{\boldsymbol{k} \sigma},
$$

the on-site Coulomb interactions

$$
H_{U}=U \sum_{i} n_{i \uparrow} n_{i \downarrow}
$$

and the exchange interactions

$$
H_{J}=\frac{1}{2} \sum_{i, j} J_{i j}\left(\boldsymbol{S}_{i} \cdot \boldsymbol{S}_{j}-\frac{1}{4} n_{i} n_{j}\right) .
$$

We have defined $\boldsymbol{S}_{i}=\frac{1}{2} \sum_{\sigma \sigma^{\prime}} c_{i \sigma}^{\dagger} \boldsymbol{\sigma}_{\sigma \sigma^{\prime}} c_{i \sigma^{\prime}}, n_{i}=\sum_{\sigma} n_{i \sigma}$, and $n_{i \sigma}=c_{i \sigma}^{\dagger} c_{i \sigma}$, where $\boldsymbol{\sigma}$ denotes the vector of Pauli matrices, and $c_{\boldsymbol{k} \sigma}$ and $c_{i \sigma}$ denote the electron operators. We define $J_{i j}=J>0$ for $\boldsymbol{R}_{j}=\boldsymbol{R}_{i} \pm \hat{c}, J_{i j}=-J_{\|}<0$ for nearest neighbor sites $(i, j)$ on the same layer, and 
$J_{i j}=0$ otherwise. Here, $\boldsymbol{R}_{i}$ and $\hat{c}$ denote the lattice vector of the site $i$ and the unit lattice vector in the $c$ direction. The interlayer antiferromagnetic exchange interaction $J$ originates from the interlayer superexchange or kinetic exchange processes of electrons. The intralayer ferromagnetic exchange interaction $J_{\|}$expresses the effect of the exchange Coulomb interaction, which is usually smaller than $U$ and $J$, but necessary to stabilize the present magnetic structure. The ferromagnetic correlation in each layer is due to $U$ and $J_{\|}$. However, twodimensional (2D) long-range order without order in the $c$-direction cannot occur due to the thermal fluctuations in the present isotropic model, however large $U$ is. Transition to the long-range order occurs only in the presence of interlayer exchange interaction $J$, and the transition to the three dimensional antiferromagnetic long-range order at $T=T_{A F}$ is the only magnetic transition.

Many examples of compounds which can be modeled by $2 \mathrm{D}$ Heisenberg ferromagnetic model with antiferromagnetic interlayer exchange interactions are summarized by a review article by Jough and Miedema [12]. For example, it was obtained from experimental data that $J / J_{\|} \approx 8 \times 10^{-3}, 3.4 \times 10^{-3}$ and $0.21, T_{A F}=13.8$, 16.8 and $18 \mathrm{~K}$, and $J_{\|} / k_{B}=18.8,5.25$ and $3.0 \mathrm{~K}$ in the compounds $\mathrm{Rb}_{2} \mathrm{CuCl}_{4}, \mathrm{CrCl}_{3}$ and $\mathrm{NaCrS}_{2}$, respectively. These compounds have the ferromagnetic shortrange order in each layer at temperatures higher than the transition temperature due to the intralayer ferromagnetic exchange interaction $J_{\|}$, and undergo the longrange order by the weak interlayer exchange interaction $J$ at the transition temperature $T_{A F}$. In many examples, the interlayer exchange interactions are antiferromagnetic and much weaker than the intralayer interaction in most cases. In our model, we also take into account the on-site Coulomb repulsion $U$ in addition to the intralayer exchange interaction $J_{\|}$to stabilize the ferromagnetic structure in each layer. Later, we consider a situation in which $U \gg J_{\|}$as an example, but it is straightforward to apply the theory to the opposite case $U \ll J_{\|}$.

It is well-known due to the Mermin-Wagner's theorem [13] that purely 2D isotropic Heisenberg model cannot exhibit any long-range order at any finite temperature. For the long-range order to be stabilized, an additional Ising type intralayer interaction or a three dimensional (interlayer) interaction is necessary. However, the former does not stabilize the present antiferromagnetic configuration in the stacking direction, as observed in $\mathrm{UPd}_{2} \mathrm{Al}_{3}$ [1] and $\mathrm{CePt}_{3} \mathrm{Si}$ [2]. Therefore, more or less, interlayer antiferromagnetic interaction must exist in the present compounds. The physical origin of the antiferromagnetic interlayer interaction is interlayer kinetic exchange or superexchange process. In the former process, the interlayer exchange interaction $J$ is written as $J \sim 4 t_{\perp}^{2} / U$, where $t_{\perp}$ denotes interlayer electron hopping energy. Since $t_{\perp}$ is expected to be small from the crystal structure, the perturbation theory to derive the above experssion of $J$ would be justified.
When we apply the Hamiltonian eq. (11) to the compound $\mathrm{UPd}_{2} \mathrm{Al}_{3}$, we should note that it has been suggested [14, 15] by thermodynamic measurements that the magnetic and superconducting transitions occur in nearly disjunct subsystems in this compound. However, even if this is true, the present theory holds if the superconducting subsystem has a similar interlayer exchange interaction, which is plausible because both of the two subsystems have a $5 f$ character and coexist in the same crystal structure. We discuss an application of the present theory taking into account the two-fluid model later.

The interaction terms can be rewritten as

$$
H_{U}+H_{J}=\frac{1}{N} \sum_{\boldsymbol{k} \boldsymbol{k}^{\prime} \boldsymbol{q}} V\left(\boldsymbol{k}, \boldsymbol{k}^{\prime}, \boldsymbol{q}\right) c_{\boldsymbol{k}+\boldsymbol{q} \uparrow}^{\dagger} c_{\boldsymbol{k} \uparrow} c_{\boldsymbol{k}^{\prime}-\boldsymbol{q} \downarrow}^{\dagger} c_{\boldsymbol{k}^{\prime} \downarrow}
$$

with

$$
\begin{aligned}
V\left(\boldsymbol{k}, \boldsymbol{k}^{\prime}, \boldsymbol{q}\right)= & U-\hat{J}(\boldsymbol{q})-\hat{J}\left(\boldsymbol{k}-\boldsymbol{k}^{\prime}+\boldsymbol{q}\right) \\
& +\hat{J}_{\|}(\boldsymbol{q})+\hat{J}_{\|}\left(\boldsymbol{k}-\boldsymbol{k}^{\prime}+\boldsymbol{q}\right),
\end{aligned}
$$

where $\hat{J}(\boldsymbol{q}) \equiv J \cos (\boldsymbol{q} \cdot \hat{c})$ and $\hat{J}_{\|}(\boldsymbol{q})$ are the Fourier transforms of the interlayer and intralayer exchange interactions, respectively. Since we have not specified the lattice structure of the layer, the expression of $\hat{J}_{\|}(\boldsymbol{q})$ is not shown, but it does not depend on $q_{z}$ and must have a peak around $\boldsymbol{q}_{\|}=\mathbf{0}$, where $\boldsymbol{q}_{\|}=\left(q_{x}, q_{y}\right)$. Similarly, the form of $\xi(\boldsymbol{k})$ also depends on the lattice structure of the layer. We have simplified it as $\xi(\boldsymbol{k})=\xi_{\|}\left(\boldsymbol{k}_{\|}\right)+\xi_{\perp}\left(k_{z}\right)$ with $\xi_{\|}\left(\boldsymbol{k}_{\|}\right)=\hbar^{2}\left|\boldsymbol{k}_{\|}\right|^{2} / 2 m^{*}-\mu$ and $\xi_{\perp}\left(k_{z}\right) \equiv-2 t_{\perp} \cos \left(k_{z} c\right)$ for convenience, where $\boldsymbol{k}_{\|}=\left(k_{x}, k_{y}\right)$. This simplification does not essentially change the qualitative results. In this paper, we examine the system in which $t_{\perp}$ and $J_{\|}$ are small. We take units with $\hbar=k_{B}=1$.

First, we describe the magnetic transition. Let us examine the spin propagator

$$
\chi\left(\boldsymbol{R}_{i}-\boldsymbol{R}_{j}, \tau\right)=-\left\langle T_{\tau} S_{i}^{z}(\tau) S_{j}^{z}(0)\right\rangle
$$

in the random phase approximation (RPA). We define the Fourier transform $\chi\left(\boldsymbol{q}, i \nu_{m}\right)$ by

$$
\chi\left(\boldsymbol{q}, i \nu_{m}\right) \equiv \sum_{i} \int_{0}^{\beta} d \tau e^{-i\left(\boldsymbol{q} \cdot \boldsymbol{R}_{i}-\nu_{m} \tau\right)} \chi\left(\boldsymbol{R}_{i}, \tau\right),
$$

where $\nu_{m} \equiv 2 \pi m T$ denotes the Matsubara frequency, and $\beta=1 / T$. If $\chi(\boldsymbol{Q}, 0)$ diverges, it indicates the phase transition to the magnetic long-range order with $\boldsymbol{Q}$. If we omit $t_{\perp}$ and $J_{\|}$, we obtain

$$
\chi\left(\boldsymbol{q}, i \nu_{m}\right)=\frac{1}{2} \frac{\chi_{0}\left(\boldsymbol{q}, i \nu_{m}\right)}{1-[U-\hat{J}(\boldsymbol{q})] \chi_{0}\left(\boldsymbol{q}, i \nu_{m}\right)},
$$

where

$$
\begin{aligned}
\chi_{0}\left(\boldsymbol{q}, i \nu_{m}\right)=- & \frac{1}{N} \sum_{\boldsymbol{k}} T \sum_{n} G_{\sigma}^{(0)}\left(\boldsymbol{k}, i \omega_{n}\right) \\
& \times G_{\sigma}^{(0)}\left(\boldsymbol{k}+\boldsymbol{q}, i \omega_{n}+i \nu_{m}\right)
\end{aligned}
$$


with the bare electron Green's function $G_{\sigma}^{(0)}$. When $t_{\perp}=$ 0 , the free susceptibility $\chi_{0}$ is expressed as

$$
\chi_{0}(\boldsymbol{q}, 0)=\rho_{0}\left(1-\operatorname{Re}\left[\sqrt{1-\left(\frac{2 k_{F \|}}{q_{\|}}\right)^{2}}\right]\right)
$$

at $T=0$, where $\rho_{0}=m^{*} a b / 2 \pi \hbar^{2}$ denotes the density of states of the 2D system. We have defined the effective mass $m^{*}$, the in-plane Fermi momentum $k_{F \|}$, and the lattice constants $a$ and $b$. The maximum $\chi\left(\boldsymbol{q}, i \nu_{m}\right)$ occurs at arbitrary $\boldsymbol{q}=\left(\boldsymbol{q}_{\|}, \pi / c\right)$ with $\left|\boldsymbol{q}_{\|}\right|<2 k_{F \|}$ and $\nu_{m}=0$. This degeneracy is removed by $J_{\|} \neq 0$, which is small but exists in practice. Hence, $\chi\left(\boldsymbol{q}, i \nu_{m}\right)$ reaches its maximum at $\boldsymbol{q}=(0,0, \pi / c) \equiv \boldsymbol{Q}$. It is easily verified by replacing $U-\hat{J}$ with $U-\hat{J}+\hat{J}_{\|}$in eq. (9). Furthermore, when we take into account $t_{\perp} \neq 0, \chi_{0}$ has a peak around $\boldsymbol{q}=\left(\boldsymbol{q}_{\|}, \pi / c\right)=\boldsymbol{Q}^{\prime}$ with $\left|\boldsymbol{q}_{\|}\right| \approx 2 k_{F \|}$, but the difference $\chi_{0}\left(\boldsymbol{Q}^{\prime}, 0\right)-\chi_{0}(\mathbf{0}, 0) \propto t_{\perp}^{2}$ is small. Therefore, when $t_{\perp} \neq 0$, we must assume $J_{\|} \neq 0$ which is small but sufficiently large for the maximum of $\chi$ to occur at $\boldsymbol{q}=\boldsymbol{Q}$, so that the magnetic order of $\boldsymbol{Q}$ is stabilized.

When these conditions are satisfied, antiferromagnetic transition occurs at a temperature which satisfies

$$
1=(U+J) \chi_{0}(\boldsymbol{Q}, 0)
$$

from eq. (9), where $\chi_{0}(\boldsymbol{Q}, 0)=\chi_{0}(\mathbf{0}, 0)=\rho_{0} /\left(e^{-\beta \mu}+1\right)$. The chemical potential $\mu$ is determined by the equation for the electron number per site $n=2 \beta^{-1} \rho_{0} \ln \left(1+e^{\beta \mu}\right)$. Thus, we obtain $\chi_{0}(\boldsymbol{Q}, 0)=\rho_{0}\left(1-e^{-\beta n / 2 \rho_{0}}\right)$. Therefore, we obtain the antiferromagnetic transition temperature

$$
T_{A F}=\frac{n}{2 \rho_{0} \ln \frac{(U+J) \rho_{0}}{(U+J) \rho_{0}-1}}
$$

to an ordered state with the wave vector $\boldsymbol{Q}=(0,0, \pi / c)$ when $(U+J) \rho_{0}>1$, while $T_{A F}=0$ otherwise.

In the antiferromagnetic phase, the electron states are affected by spontaneous staggered magnetization. We define $A$ and $B$ sublattices (sublayers) whose majority spins are up and down, respectively. We write the electron operators as $a_{i \sigma}$ and $b_{j \sigma}$ for $i \in A$ and $j \in B$. Therefore, we have

$$
\begin{aligned}
& n_{i \sigma}^{A}=\left\langle a_{i \sigma}^{\dagger} a_{i \sigma}\right\rangle=\frac{n}{2}+\sigma m, \\
& n_{j \sigma}^{B}=\left\langle b_{j \sigma}^{\dagger} b_{j \sigma}\right\rangle=\frac{n}{2}-\sigma m,
\end{aligned}
$$

where $\left\langle S_{i}^{z}\right\rangle=-\left\langle S_{j}^{z}\right\rangle=m$ for $i \in A$ and $j \in B$. We have defined $\sigma=+1$ and -1 in equations, which correspond to $\sigma=\uparrow$ and $\downarrow$ in suffixes, respectively. Corrections to the kinetic energy due to $H_{U}+H_{J}$ are taken into account by the mean field approximation as

$$
H_{\mathrm{MF}}=-\sum_{\boldsymbol{k} \sigma}^{\prime} \sigma h_{M F}\left[a_{\boldsymbol{k} \sigma}^{\dagger} a_{\boldsymbol{k} \sigma}-b_{\boldsymbol{k} \sigma}^{\dagger} b_{\boldsymbol{k} \sigma}\right]
$$

with $h_{M F}=\left(U+J+z_{\|} J_{\|} / 2\right) m$, where $z_{\|}$denotes the number of nearest neighbor sites in the layer, and the summation $\sum_{k}^{\prime}$ is carried out over the half Brillouin zone. Therefore, the total kinetic energy term $\tilde{H}_{0} \equiv H_{0}+H_{\mathrm{MF}}$ is written as

$$
\begin{aligned}
\tilde{H}_{0}=\sum_{\boldsymbol{k} \sigma}^{\prime}[ & \xi_{\| \sigma}^{A} a_{\boldsymbol{k} \sigma}^{\dagger} a_{\boldsymbol{k} \sigma}+\xi_{\| \sigma}^{B} b_{\boldsymbol{k} \sigma}^{\dagger} b_{\boldsymbol{k} \sigma} \\
& \left.+\xi_{\perp}\left(a_{\boldsymbol{k} \sigma}^{\dagger} b_{\boldsymbol{k} \sigma}+b_{\boldsymbol{k} \sigma}^{\dagger} a_{\boldsymbol{k} \sigma}\right)\right]
\end{aligned}
$$

where $\xi_{\| \sigma}^{A}=\xi_{\|}-\sigma h_{M F}$ and $\xi_{\| \sigma}^{B}=\xi_{\|}+\sigma h_{M F}$. The mean field approximation is consistent with the RPA, which we have used to derive $T_{A F}$, although we have neglected $J_{\|}$ in eq. (13).

Now, let us examine superconductivity. We will show its formulation in the antiferromagnetic phase, but it is immediately reduced to that in the paramagnetic phase by putting $m=0$. The exchange interaction $H_{J}$ contributes to pairing interaction, while it causes the antiferromagnetic transition and creates the exchange field. We rewrite $H_{J}$ as

$$
H_{J}=-\sum_{i \in A, j \in B} J_{i j} \Psi_{i j}^{(\mathrm{s})} \Psi_{i j}^{(\mathrm{s})},
$$

where $\Psi_{i j}^{(\mathrm{s})}=2^{-1 / 2}\left(a_{i \uparrow} b_{j \downarrow}-a_{i \downarrow} b_{j \uparrow}\right)$. The statistical average $\left\langle\Psi_{i j}^{(\mathrm{s})}\right\rangle$ is the order parameter of interlayer spinsinglet pairing. Here, we have neglected $J_{\|}$, since it does not have an important effect on superconductivity if it is small. In eq. (17), it is found that $J$ contributes only to spin-singlet pairing as an attractive interaction in its first order. In the BCS approximation, eq. (17) is written as

$$
H_{J} \approx \sum_{\boldsymbol{k} \sigma} \sigma\left[\Delta(\boldsymbol{k}) a_{\boldsymbol{k} \sigma} b_{-\boldsymbol{k}-\sigma}+\text { h.c. }\right]
$$

with the order parameter

$$
\Delta(\boldsymbol{k})=-\frac{1}{2 N} \sum_{\boldsymbol{k}^{\prime} \sigma^{\prime}}^{\prime} \sigma^{\prime} \hat{J}\left(\boldsymbol{k}-\boldsymbol{k}^{\prime}\right)\left\langle b_{-\boldsymbol{k}^{\prime}-\sigma^{\prime}}^{\dagger} a_{\boldsymbol{k}^{\prime} \sigma^{\prime}}^{\dagger}\right\rangle .
$$

Therefore, we obtain

$$
\Delta(\boldsymbol{k})=\Delta_{0} \cos \left(k_{z} c\right)
$$

with

$$
\Delta_{0}=-\frac{J}{2 N} \sum_{\boldsymbol{k}^{\prime} \sigma^{\prime}}^{\prime} \sigma^{\prime} \cos \left(k_{z}^{\prime} c\right)\left\langle b_{-\boldsymbol{k}^{\prime}-\sigma^{\prime}}^{\dagger} a_{\boldsymbol{k}^{\prime} \sigma^{\prime}}^{\dagger}\right\rangle .
$$

In the same approximation, the on-site Coulomb interaction $H_{U}$ is ineffective for anisotropic superconductivity.

It has been proposed that in the higher order of $J$, the pairing interaction is enhanced by the exchange of magnons [16]. This mechanism has also been examined in the compound $\mathrm{UPd}_{2} \mathrm{Al}_{3}[17,18,19,20$. However, since the spin fluctuations are weak at temperatures much lower than the antiferromagnetic transition temperature $\left(T \lesssim T_{A F} / 7\right)$, the pairing interaction mediated by the magnons is weak [16]. Hence, in this paper, we neglect 
them in comparison to the direct pairing interaction described in eqs. (17) and (18). The present direct pairing interaction is not mediated by the magnons. In a broader sense, however, one may regard the present pairing interaction as mediated by the spin fluctuations, because the superexchange interactions are derived from the virtual process of electrons, with which their spins correlate.

When $J=0$, the present model is reduced to the quasi2D Hubbard model. In the perturbation theory based on it, it was shown that antiferromagnetic fluctuations induce intralayer singlet pairing near the antiferromagnetic phase 21]. However, the present system is ferromagnetic in each layer. In the absence of $J$, the propagator of the fluctuations $\chi(\boldsymbol{q})$ has a broad peak around $\boldsymbol{q}_{\|} \approx \mathbf{0}$ as we can see in eq. (9), in contrast to the sharp peak in the antiferromagnetic case, where the Fermi-surface nesting occurs. Therefore, the pairing interaction is not strongly enhanced by the spin fluctuation unless $U$ is large. Nisikawa and Yamada 22 examined the $\mathrm{UPd}_{2} \mathrm{Al}_{3}$ on the basis of a 2D Hubbard model taking into account the lattice structure, although they did not examine interlayer pairing. In the presence of interlayer interactions, $t_{\perp}$ and $J$, the spin fluctuations will enhance the interlayer singlet pairing interaction.

From eqs. (16) and (18), we obtain

$$
H=\sum_{\boldsymbol{k}} \psi_{\boldsymbol{k}}^{\dagger} \hat{M} \psi_{\boldsymbol{k}}
$$

where we have defined $\psi_{\boldsymbol{k}}^{\dagger} \equiv\left(a_{\boldsymbol{k} \uparrow}^{\dagger} b_{\boldsymbol{k} \uparrow}^{\dagger} a_{-\boldsymbol{k} \downarrow} b_{-\boldsymbol{k} \downarrow}\right)$ and

$$
\hat{M}(\boldsymbol{k}) \equiv\left(\begin{array}{cccc}
\xi_{\| \uparrow}^{A} & \xi_{\perp} & 0 & -\Delta^{*} \\
\xi_{\perp} & \xi_{\| \uparrow}^{B} & -\Delta^{*} & 0 \\
0 & -\Delta & -\xi_{\| \downarrow}^{A} & -\xi_{\perp} \\
-\Delta & 0 & -\xi_{\perp} & -\xi_{\| \downarrow}^{B}
\end{array}\right) .
$$

The Green's function is defined in matrix form by

$$
\hat{G}(\boldsymbol{k}, \tau)=-\left\langle T_{\tau} \psi_{\boldsymbol{k}}(\tau) \psi_{\boldsymbol{k}}^{\dagger}\right\rangle .
$$

From the equation of motion, we obtain

$$
\hat{G}\left(\boldsymbol{k}, i \omega_{n}\right)=\left[i \omega_{n} \hat{I}-\hat{M}(\boldsymbol{k})\right]^{-1},
$$

where $\hat{I}$ denotes the $2 \times 2$ unit matrix. We obtain the quasi-particle energies, $\pm E_{A}(\boldsymbol{k})$ and $\pm E_{B}(\boldsymbol{k})$, where $E_{A}^{2}=E^{2}+F, E_{B}^{2}=E^{2}-F, E^{2}=\xi_{\|}^{2}+\xi_{\perp}^{2}+h_{M F}^{2}+|\Delta|^{2}$ and $F=2\left[\xi_{\|}^{2}\left(\xi_{\perp}^{2}+h_{M F}^{2}\right)+|\Delta|^{2} \xi_{\perp}^{2} / 2\right]^{1 / 2}$. If we define $G_{i j}$ as the $(i, j)$ component of the matrix $\hat{G}$, we have

$$
\begin{aligned}
& \left\langle b_{\boldsymbol{k}^{\prime} \uparrow}^{\dagger} a_{-\boldsymbol{k}^{\prime} \downarrow}^{\dagger}\right\rangle=G_{32}\left(\boldsymbol{k}^{\prime}, \tau=-0\right), \\
& \left\langle b_{-\boldsymbol{k}^{\prime} \downarrow}^{\dagger} a_{\boldsymbol{k}^{\prime} \uparrow}^{\dagger}\right\rangle=-G_{41}\left(\boldsymbol{k}^{\prime}, \tau=-0\right) .
\end{aligned}
$$

Therefore, we obtain the gap equation

$$
\Delta(\boldsymbol{k})=\frac{1}{4 N} \sum_{\boldsymbol{k}^{\prime}}^{\prime} \hat{J}\left(\boldsymbol{k}-\boldsymbol{k}^{\prime}\right) \sum_{X=A, B} \frac{\tanh \frac{E_{X}\left(\boldsymbol{k}^{\prime}\right)}{2 T}}{2 E_{X}\left(\boldsymbol{k}^{\prime}\right)} \Delta\left(\boldsymbol{k}^{\prime}\right),
$$

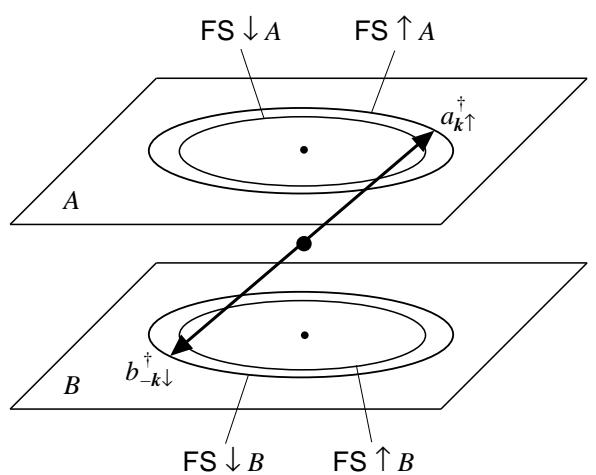

FIG. 1: Schematic figure of the Fermi surfaces of $\uparrow$ and $\downarrow$ electrons in the $A$ and $B$ layers, and interlayer spin-singlet pairing. The abbreviation FS $\sigma X$ denotes the Fermi surface of the spin $\sigma$ electrons on the $X$ sublattice, where $\sigma=\uparrow, \downarrow$ and $X=A, B$. The splits of the Fermi surfaces of the $\uparrow$ and $\downarrow$ spin electrons do not affect interlayer spin-singlet pairing.

In the limit of $\Delta_{0} \rightarrow 0$, we have $E_{X}=\left|\xi_{X}\right|$, where $\xi_{A}=$ $\xi_{\|}+\delta_{\perp}, \xi_{B}=\xi_{\|}-\delta_{\perp}$, and $\delta_{\perp}=\sqrt{h_{M F}^{2}+\xi_{\perp}^{2}}$. Therefore, we obtain the equation for $T_{c}$

$$
1=\frac{J}{4 N} \sum_{\boldsymbol{k}^{\prime}}^{\prime} \cos ^{2}\left(k_{z}^{\prime} c\right) \sum_{X=A, B} \frac{\tanh \left[\xi_{X}\left(\boldsymbol{k}^{\prime}\right) / 2 T_{c}\right]}{2 \xi_{X}\left(\boldsymbol{k}^{\prime}\right)} .
$$

It is easily verified that the right hand side of eq. (28) exhibits a logarithmic divergence in the limit of $T \rightarrow 0$, irrespective of the value of the exchange field $h_{M F}$. Logarithmic divergence, which results from the Fermi-surface instability, is crucial for the occurrence of superconductivity. Since the divergence occurs even if $h_{M F}$ is very large, the existence of a large local magnetic moment as observed in $\mathrm{UPd}_{2} \mathrm{Al}_{3}[3]$ does not deny the occurrence of superconductivity in the electrons which have the present magnetic structure. It is interesting that superconductivity is not influenced by large spin polarization in each layer, although such polarization creates the strong exchange field on the electrons responsible to superconductivity. It is well-known that in ferromagnets, strong exchange field suppresses superconductivity by Pauli paramagnetic pair-breaking effect.

This result can easily be verified as follows. The present superconductivity is due to singlet pairing of $a_{\boldsymbol{k} \sigma}$ and $b_{-\boldsymbol{k}-\sigma}$ electrons, i.e., spin $\sigma$ electrons on $A$ sublattice and spin $-\sigma$ electrons on $B$ sublattice. When we define the Fermi surface of each sublattice, the magnitudes of the Fermi momenta of those electrons are equal, irrespective of the magnitude of the Zeeman splitting due to the exchange field in each layer, as schematically shown in Fig. 1 Therefore, the present pair states are not influenced by the magnetic moments.

Needless to say, interlayer pairing does not mean that the coherence length in the $c$-direction $\xi_{0 \perp}$ is on the order of the layer spacing. In the present system, $\xi_{0 \perp}$ is on 
the order of $v_{F \perp} / \Delta_{0}$, where $v_{F \perp}$ and $\Delta_{0}$ denote Fermi velocity in the $c$-direction and the scale of magnitude of the order parameter at $T=0$, respectively. We obtain $\xi_{0 \perp} \gg c$, if $t_{\perp} \gg \Delta_{0}$.

When $t_{\perp}$ is negligible, the transition temperature $T_{c}$ is obtained as follows. Since $\delta_{\perp}=\left|h_{M F}\right|$, we can integrate $\cos ^{2}\left(k_{z}^{\prime} c\right)$ with respect to $k_{z}^{\prime}$ first in eq. (28). Unless $\delta_{\perp}>$ $\mu$, the density of states is constant when $t_{\perp} \approx 0$. The $\boldsymbol{k}_{\|}$integral is approximated by the $\xi_{\|} \pm h_{M F}$ integrals with an effective cutoff energy $W_{c}$, which is on the order of the band width. More explicitly, it is expressed as $W_{c}=\left[\left(W-\mu-h_{M F}\right)\left(W-\mu+h_{M F}\right)\left(\mu+h_{M F}\right)(\mu-\right.$ $\left.\left.h_{M F}\right)\right]^{1 / 4}$, where $W$ and $\mu$ denote the band width and the chemical potential measured from the bottom of the band, respectively. Carrying out the integral, we obtain

$$
T_{c}=1.13 W_{c} e^{-2 / J \rho_{0}} .
$$

Here, it is found that $T_{c}$ is not influenced by the spontaneous staggered magnetization $g \mu_{B} m$, as expected from the above argument.

Figure 2 depicts the antiferromagnetic transiton temperature $T_{A F}$ and the superconducting transition temperature $T_{c}$ scaled by $n / \rho_{0}$ and $W_{c}$, respectively. These scales have the same orders of magnitude, i.e., $\rho_{0} \sim$ $1 / W_{c}$. It is found that, as the order of the magnitudes, the experimental results in $\mathrm{UPd}_{2} \mathrm{Al}_{3}, T_{A F}=14.3 \mathrm{~K}$ and $T_{c}=2.0 \mathrm{~K}$ could be explained within the present mechanism, if the effective band width is on the order of $W=10 \sim 100 \mathrm{~K}$, which is realistic for heavy fermion systems.

Figure 3 shows the values of $J \rho_{0}$ and $U \rho_{0}$ for a given ratio of $\alpha \equiv\left(T_{A F} \rho_{0} / n\right) /\left(T_{c} / W_{c}\right)=T_{A F} / T_{c} \times W_{c} \rho_{0} / n$. If we consider $W_{c} \rho_{0} / n \sim 1$, the experimental data $T_{A F}=$ $14.3 \mathrm{~K}$ and $T_{c}=2.0 \mathrm{~K}$ give $\alpha \sim 7$. Therefore, the experimental value of ratio $\alpha$ can be reasonably reproduced for moderate values of the coupling constants $J$ and $U$. We will compare the theoretical and experimental results more closely below. We obtain $T_{A F}>T_{c}$ or $T_{A F}=0$, except in a very small region of the phase diagram.

We note that the resultant singlet order parameter is invariant under rotation in spin space. The rotational transformation is made by

$$
R(\theta)=\exp \left[i \frac{\theta}{2} \sigma_{y}\right]=\cos \frac{\theta}{2}+i \sigma_{y} \sin \frac{\theta}{2} .
$$

The electron operators in the rotated space are defined by

$$
\left(\begin{array}{c}
\tilde{c}_{\boldsymbol{k} \uparrow}^{\dagger} \\
\tilde{c}_{\boldsymbol{k} \downarrow}^{\dagger}
\end{array}\right)=R(\theta)\left(\begin{array}{c}
c_{\boldsymbol{k} \uparrow}^{\dagger} \\
c_{\boldsymbol{k} \downarrow}^{\dagger}
\end{array}\right),
$$

where $c_{\boldsymbol{k} \sigma}=a_{\boldsymbol{k} \sigma}$ or $b_{\boldsymbol{k} \sigma}$. It is easily verified that

$$
\sum_{\sigma} \sigma\left\langle b_{-\boldsymbol{k}-\sigma}^{\dagger} a_{\boldsymbol{k} \sigma}^{\dagger}\right\rangle=\sum_{\sigma} \sigma\left\langle\tilde{b}_{-\boldsymbol{k}-\sigma}^{\dagger} \tilde{a}_{\boldsymbol{k} \sigma}^{\dagger}\right\rangle
$$

In addition, the present pairing interaction is rotationally invariant, i.e., $\hat{J}\left(\boldsymbol{k}, \boldsymbol{k}^{\prime}\right)$ does not have spin suffixes.

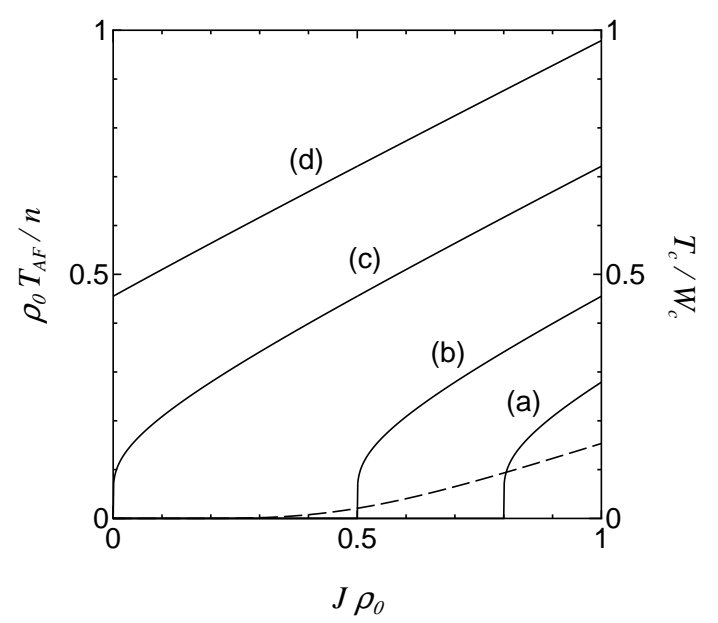

FIG. 2: The transition temperatures as functions of $J \rho_{0}$. The solid curves (a) - (d) show the results of $\rho_{0} T_{A F} / n$ for $U \rho_{0}=0.2,0.5,1.0$, and 1.5 , respectively. The dashed curve shows the results of $T_{c} / W_{c}$.

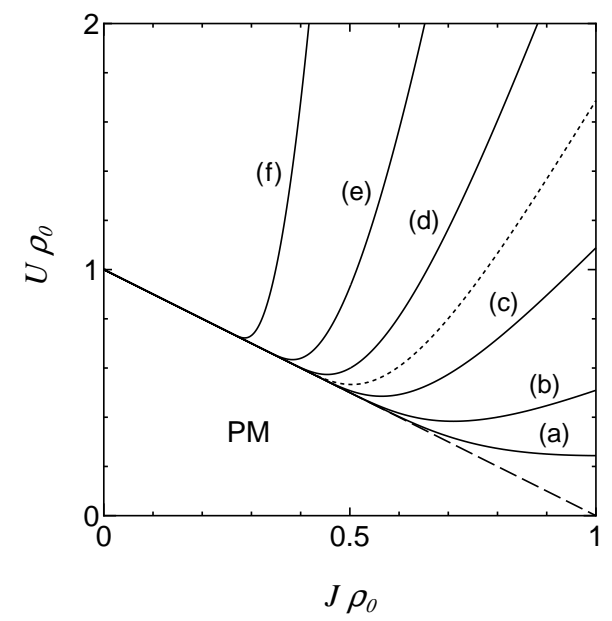

FIG. 3: Contour lines for various ratios $T_{A F} / T_{c}$ in $J-U$ plane. The solid curves (a) - (f) are the countour lines for $\alpha=2,3,5,10,20$, and 100, respectively, where $\alpha \equiv$ $\left(T_{A F} \rho_{0} / n\right) /\left(T_{c} / W_{c}\right)=T_{A F} / T_{c} \times W_{c} \rho_{0} / n$. The dotted curve is that of $\alpha=7$, which corresponds to the experimental result of $\mathrm{UPd}_{2} \mathrm{Al}_{3}$. The dashed curve shows the phase boundary between the antiferromagnetic and paramagnetic (PM) phases at $T=0$.

Therefore, $\Delta(\boldsymbol{k})$ given by eq. (19) is isotropic in spin space, irrespective of the direction of the magnetic order. In fact, the resultant gap equations (27) and (28) are invariant under spin rotation.

These results may explain the experimental data of the muon spin rotation measurements in $\mathrm{UPd}_{2} \mathrm{Al}_{3}$ [23], in which it was observed that the London penetration depth and the magnetic susceptibility reduction below 
$T_{c}$ are essentially isotropic, while the total susceptibility remains strongly anisotropic. In the present mechanism, not only the singlet nature but also the rotational invariance of the pairing interaction play essential roles in the spin isotropy of $\Delta(\boldsymbol{k})$. In contrast, in the "magnetic exciton" mechanism, the pairing interaction and the order parameter are anisotropic if the magnetic system is anisotropic.

The compound $\mathrm{CePt}_{3} \mathrm{Si}$ is another candidate for the present pairing mechanism, although the ratio of the transition temperatures $T_{A F} / T_{c}$ is much smaller than that in $\mathrm{UPd}_{2} \mathrm{Al}_{3}$. In contrast to $\mathrm{UPd}_{2} \mathrm{Al}_{3}$, the critical field largely exceeds the Pauli paramagnetic limit $H_{P}$ estimated by the simplified formula $H_{\mathrm{P}} \approx 1.86[\mathrm{~T} / \mathrm{K}] \times$ $T_{\mathrm{c}}[\mathrm{K}] \approx 1.4 \mathrm{~T}$ in $\mathrm{CePt}_{3} \mathrm{Si}[4]$. If the present mechanism of singlet pairing is realized in $\mathrm{CePt}_{3} \mathrm{Si}$, the large critical field cannot be attributed to equal spin pairing. It can be explained by an effect of exchange field created by coexisting antiferromagnetic long-range order, which reduces the Pauli paramagnetic pair-breaking effect [24]. It is still controversial whether dominant pairing in $\mathrm{CePt}_{3} \mathrm{Si}$ is of singlet or triplet. Recent NMR data suggests that the gap function may have some novel structure [25]. Thermal transport measurements have suggested that the order parameter has line nodes [26], which is consistent with the present theory. It is known that the compound $\mathrm{CePt}_{3} \mathrm{Si}$ does not have inversion symmetry. The Rashba interaction [27] has been examined to include it. Yip predicted that the Knight shift vanishes in the superconductors with strong Rashba interaction. 28]

For more close comparison with the experimental data of $\mathrm{UPd}_{2} \mathrm{Al}_{3}$, we consider a two-fluid model [14, 15]. In order to take into account the model, we assume two different renormalization factors $Z_{s}$ and $Z_{m}$ for electrons responsible to superconductivity and antiferromagnetic order, respectively. We should note that in actuality there are strong renormalization effects in the heavy fermion system, not only in the density of states, but also in the vertex corrections. It is easily verified by diagramatical consideration that in terms of $Z_{s}$ and $Z_{m}$, the electron mass $m_{0}$, the density of states $\rho_{0}$, the band width $W$, and the coupling constants $J$ and $U$ are modified as $\tilde{m}_{a}=Z_{a} m_{0}, \tilde{\rho}_{a}=Z_{a} \rho_{0}, \tilde{W}_{a}=W / Z_{a}$ $\tilde{J}_{a}=J / Z_{a}^{2}$ and $\tilde{U}_{a}=J / Z_{a}^{2}$, respectively, where $a=$ $s, m$. The experimental value $\gamma_{1}=115 \mathrm{~mJ} / \mathrm{K}^{2} \mathrm{~mol}$ [14] gives $\tilde{\rho}_{s}=1 / 476 \mathrm{~K}^{-1}$. Equation (29) is rewritten as $T_{c}=1.13 W_{c} e^{-1 / \tilde{\lambda}}$ with $\tilde{\lambda}=\tilde{J}_{s} \tilde{\rho}_{s}, \rho_{s} \sim 1 / \tilde{W}_{s}$, and $W_{c}=\tilde{W}_{s} / 2$. Hence, $T_{c}=2.2 \mathrm{~K}$ gives $\tilde{J}_{s}=173 \mathrm{~K}$. The antiferromagnetic transition temperature of eq. (13) is written as

$$
T_{A F}=\frac{n}{2 \tilde{\rho}_{m} \ln \frac{\left(\tilde{U}_{m}+\tilde{J}_{m}\right) \tilde{\rho}_{m}}{\left(\tilde{U}_{m}+\tilde{J}_{m}\right) \tilde{\rho}_{m}-1}} .
$$

Here, we simply put $n=2$ for order estimations. From the values of $\tilde{J}_{s}$ and $\tilde{\rho}_{s}$ estimated above, the values of $\tilde{J}_{m}$ and $\tilde{\rho}_{m}$ can be obtained, if the ratio $Z_{m} / Z_{s}$ is known. Therefore, we only need the values of $Z_{m} / Z_{s}$ and $\tilde{U}_{m}$ for estimation of $T_{A F}$. However, since they are not known for $\mathrm{UPd}_{2} \mathrm{Al}_{3}$ at the present, we need to assume them. In the assumption, we require that $\tilde{W}_{m}$ is smaller than $T_{A F}$ consistently with the observation of a large local magnetic moment in $\mathrm{UPd}_{2} \mathrm{Al}_{3}$ [14]. Physically, it is also plausible that $\tilde{U}_{m}$ is not much larger than $T_{A F}$ but larger than $\tilde{W}_{m}$. As an example, let us assume that $Z_{m} / Z_{s}=30$, which gives $\tilde{W}_{m} \approx 8 \mathrm{~K} \ll T_{A F}$ and $\tilde{J}_{m} \approx 0.19 \mathrm{~K}$. In this case, if we assume $\tilde{U}_{m}=20 \mathrm{~K}$ and $30 \mathrm{~K}$ as examples, we obtain $T_{A F} \approx 10 \mathrm{~K}$ and $21 \mathrm{~K}$, respectively. As another example, we assume $Z_{m} / Z_{s}=20$, which gives $\tilde{W}_{m} \approx 12 \mathrm{~K} \ll T_{A F}$ and $\tilde{J}_{m} \approx 0.43 \mathrm{~K}$. In this case $\tilde{U}_{m}=30 \mathrm{~K}$ and $40 \mathrm{~K}$ give $T_{A F} \approx 16 \mathrm{~K}$ and $27 \mathrm{~K}$, respectively. The values $\tilde{J}_{m} \approx 0.19 \mathrm{~K}$ and $0.43 \mathrm{~K}$ obtained in these examples are not outrageous as energy parameters in real materials. In fact, $J \approx 0.15 \mathrm{~K}$ and $0.63 \mathrm{~K}$ were obtained from experimental data in $\mathrm{Rb}_{2} \mathrm{CuCl}_{4}$ and $\mathrm{NaCrS}_{2}$, respectively 12. Here, $J$ was not estimated as the bare parameter but estimated as the dressed (observed) parameter like $\tilde{J}_{m}$. Although our estimations are crude, the values of $T_{A F}$ obtained above are on the same order of the experimental result $T_{A F}=14.3 \mathrm{~K}$. Therefore, we find that the present mechanism reproduces consistent orders of magnitudes of $T_{c}$ and $T_{A F}$ for appropriate values of $Z_{m} / Z_{s}$ and $\tilde{U}_{m}$.

The result that $T_{A F}>T_{c}$ except for in a very limited region unless $T_{A F} \neq 0$ can be explained physically as follows. In the present mechanism, both interlayer antiferromagnetic long-range order and interlayer singlet superconductivity are induced by interlayer antiferromagnetic exchange interaction. However, strong on-site repulsion contributes to stabilization of the ferromagnetic structure in each layer, while it does not contribute to interlayer singlet pairing. Therefore, the magnetic transition occurs at a higher temperature than the superconducting transition temperature unless the on-site $U$ is negligibly small.

Interlayer pairing has been studied by many authors 29]. In this paper, we have examined the magnetic mechanism of the pairing interactions for interlayer singlet pairing, when the electrons are on the magnetic layers. However, irrespective of the pairing mechanism, interlayer pairing of the present type seems to be the most favorable, apart from equal spin pairing, when the present type of antiferromagnetic long-range order coexists. Other pairing states, such as intralayer singlet pairing, are strongly suppressed by the splitting of the Fermi-surfaces of the electrons with up and down spins due to the antiferromagnetic moment. Even in the twofluid model, the exchange field must be induced on the electrons responsible to superconductivity.

In conclusion, in antiferromagnets with the magnetic order of the wave vector $\boldsymbol{Q}=(0,0, \pi / c)$, magnetic interactions may induce the superconductivity of interlayer spin-singlet pairing, the order parameter of which has a horizontal line node. It was found that superconductivity and an antiferromagnetic long-range order with large localized magnetic moments $m$ can coexist, and that $T_{c}$ 
is not influenced by the magnitude of $m$. It was also found that $T_{A F}>T_{c}$ in most cases, unless $T_{A F}=0$. The present model may describe an essential aspect of antiferromagnetic heavy fermion superconductors, such as $\mathrm{UPd}_{2} \mathrm{Al}_{3}$ and $\mathrm{CePt}_{3} \mathrm{Si}$. The orders of the magnitude of $T_{A F}$ and $T_{c}$ and their ratio $T_{A F} / T_{c} \approx 3 \sim 7$ can be reproduced by assuming moderate parameter values. The resultant order parameter is consistent with the observations mentioned above [5, 6, 7, 8, 23, 26].

The author wishes to thank Y. Matsuda for giving their preprint on $\mathrm{UPd}_{2} \mathrm{Al}_{3}$ and for useful discussions. This work was partially supported by the Ministry of Education, Science, Sports and Culture of Japan, Grant-in-Aid for Scientific Research (C), No.16540320, 2004.
[1] H. Kita, A. Donni, Y. Endoh, K. Kakurai, N. Sato, and T. Komatsubara, J. Phys. Soc. Jpn. 63, 726 (1994).

[2] N. Metoki, K. Kaneko, T.D. Matsuda, A. Galatanu, T. Takeuchi, S. Hashimoto, T. Ueda, R. Settai, Y. Onuki, and N. Bernhoeft, J. Phys. Condens. Matter 16, L207 (2004).

[3] C. Geibel, C. Schank, S. Thies, H. Kitazawa, C.D. Bredl, A. Bohm, M. Rau, A. Grauel, R. Caspry, R. Helfrich, U. Ahlheim, G. Weber, and F. Steglich, Z. Phys. B 84, 1 (1991); A. Krimmel, P. Fisher, B. Roessli, H. Maletta, C. Geibel, C. Shank, A. Grauel, A. Loidl, and F. Steglich, Z. Phys. B 86, 161 (1992).

[4] E. Bauer, G. Hilscher, H. Michor, Ch. Paul, E.W. Scheidt, A. Gribanov, Yu. Seropegin, H. Noel, M. Sigrist, and P. Rogl, Phys. Rev. Lett. 92, 027003 (2004).

[5] H. Tou, Y. Kitaoka, K. Asayama, C. Geibel, C. Schank, and F. Stegilch, J. Phys. Soc. Jpn. 64, 725 (1995).

[6] A. Amato, R. Feyerherm, F.N. Gygax, A. Schenck, M. Weber, R. Caspary, P. Hellmann, C. Schank, C. Geibel, F. Steglich, D.E. MacLaughlin, E.A. Knetsch, and R.H. Heffner, Europhys. Lett. 19, 127 (1992).

[7] J. Hessert, M. Huth, M. Jourdan, H. Adrian, C.T. Rieck, and K. Scharnberg, Physica B 230-232, 373 (1997).

[8] T. Watanabe, K. Izawa, Y. Kasahara, Y. Haga, Y. Onuki, P. Thalmeier, K. Maki, and Y. Matsuda, Phys. Rev. B 70, 184502 (2004).

[9] See, for example, A. V. Andreev, A.I. Buzdin, and R.M. Osgood III, Phys. Rev. B 43, 10124 (1991); M. Houzet, A. Buzdin, and M.L. Kulic, Phys. Rev. B 64, 184501 (2001); A.Yu. Aladyshkin, A.I. Buzdin, A.A. Fraerman, A.S. Mel'nikov, D.A. Ryzhov, and A.V. Sokolov, Phys. Rev. B 68, 184508 (2003); H. Shimahara and S. Hata, Phys. Rev. B 62, 14541 (2000); T. Champel and M. Eschrig, Phys. Rev. B 71, 220506 (2005).

[10] Yu.V. Kopaev, Fizika Tverdogo Tela, 7, 2907 (1965), [Sov. Phys. Solid State 7, 2360 (1966)].

[11] A.I. Buzdin and A.S. Mel'nikov, Phys. Rev. B 67, 020503 (2003).

[12] L.J. de Jough and A.R. Miedema, Advances in Phys. 23, 1 (1974), and references therein.

[13] N.D. Mermin and H. Wagner, Phys. Rev. Lett. 17, 1307
(1966).

[14] R. Caspary, P. Hellmann, M. Keller, G. Sparn, C. Wassilew, R. Kohler, C. Geibel, C. Schank, and F. Steglich, N.E. Phillips, Phys. Rev. Lett. 71, 2146 (1993).

[15] J. Gen, P. Coleman, and N. Andrei, Phys. Rev. Lett. 68, 3476 (1992).

[16] H. Shimahara, J. Phys. Soc. Jpn. 63, 1861 (1994).

[17] N.K. Sato, N. Aso, K. Miyake, R. Shiina, P. Thalmeier, G. Varelogiannis, C. Geibel, F. Steglich, P. Fulde, and T. Komatsubara Nature 410340 (2001).

[18] The magnetic excitations (i.e., the magnons) are called "magnetic excitons" in ref. [17], for some reason.

[19] K. Miyake and N.K. Sato, Phys. Rev. B 63, 052508 (2001).

[20] The exchange interactions $J(\boldsymbol{q})$ considered in ref. 19] are those between the itinerant electrons and the localized spins as $J_{K}$ in ref. [16], while our $\hat{J}(\boldsymbol{q})$ in the present paper are those between the itinerant electrons on adjacent layers.

[21] H. Shimahara and S. Takada, J. Phys. Soc. Jpn. 57, 1044 (1988); H. Shimahara, J. Phys. Soc. Jpn. 58, 1735 (1989).

[22] Y. Nisikawa and K. Yamada, J. Phys. Soc. Jpn. 71, 237 (2002).

[23] R. Feyerherm, A. Amato, F.N. Gygax, A. Schenck, C. Geibel, F. Steglich, N. Sato, and T. Komatsubara, Phys. Rev. Lett. 73, 1849 (1994).

[24] H. Shimahara, J. Phys. Soc. Jpn. 73, 2635 (2004).

[25] M. Yogi, Y. Kitaoka, S. Hashimoto, T. Yasuda, R. Settai, T.D. Matsuda, Y. Haga, Y. Onuki, P. Rogl, and E. Bauer, Phys. Rev. Lett. 93, 027003 (2004).

[26] K. Izawa, Y. Kasahara, Y. Matsuda, K. Behnia, T. Yasuda, R. Settai, and Y. Onuki, Phys. Rev. Lett. 94, 197002 (2005).

[27] E.I. Rashba, Fiz. Tverd. Tela (Leningrad) 2, 1224 (1960) [Sov. Phys. Solid State 2, 1109 (1960)].

[28] S.K. Yip, Phys. Rev. B 65, 144508 (2002).

[29] See, for example, R.A. Klemm and S.H. Liu, Phys. Rev. B 44, 7526 (1991); S. Kettemann and K.B. Efetov, Phys. Rev. B 46, 8515 (1992); S.H. Liu and R.A. Klemm, Phys. Rev. B 48, 4080 (1993); A.K. Rajagopal and S.S. Jha, Phys. Rev. B bf 47, 2780 (1993). 